\title{
A GENERAL FORM \\ OF THE SUSPENSION BRIDGE CATENARY
} BY IRA FREEMAN

1. Introduction. The form of the curve assumed by a uniform chain or string hanging freely between two supports was first investigated by Galileo, who erroneously determined it to be a parabola; Jungius detected Galileo's error, but the true form was not discovered until 1691, when James Bernoulli published it as a problem in the Acta Eruditorum. He also considered the cases when the chain was (1) of variable density, (2) extensible, (3) acted upon at each point by a force directed to a fixed center.

These curves attracted much attention, and were discussed by John Bernoulli, Leibnitz, Huygens, David Gregory and others. A review of the literature shows, however, that in the problem of the inextensible string acted upon by gravitational forces, only two special cases are considered:- the case where there is simply a string of uni-form linear density, and the case of a string of inappreciable

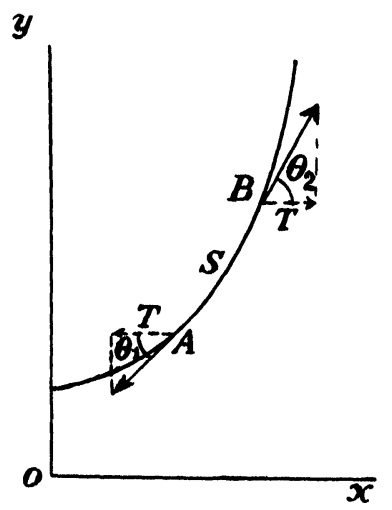
weight supporting a uniform hori-

zontal load. Moreover, the modern text-books in the subject appear to shun a more general case, and content themselves with presenting only the two very special cases mentioned.

The object of the present paper is to consider what happens when a cable of appreciable uniform linear density supports a uniform horizontal load, conditions that are very nearly realized in the suspension-bridge. The texts solve the special cases by the solution of two first-order 
differential equations, the first of which gives the intrinsic equation of the curve. The following method uses one second-order differential equation, and does not involve the intrinsic equation as an intermediate step.

2. Densities Different and not Zero. Both the cable and the bridge platform have uniform linear densities which are appreciable, and, in general, different.

Let $\alpha$ be the weight per unit length of the cable, and let $\beta$ represent that of the bridge platform. If any arc $s$ is in equilibrium, the horizontal component of the tensions, at the extremities of the arc, will be the same constant which we may call $T$. The difference of the vertical components of the extreme tensions will equal the total weight on the are; that is, we shall have

$$
T\left(\tan \theta_{2}-\tan \theta_{1}\right)=\alpha s+\beta x .
$$

If $s$ is small, this becomes

$$
T \frac{d^{2} y}{d x^{2}}=\alpha \frac{d s}{d x}+\beta,
$$

since

$$
\tan \theta_{2}-\tan \theta_{1}=\frac{d}{d x}\left(\frac{d y}{d x}\right) d x .
$$

But since

$$
\left(\frac{d s}{d x}\right)^{2}=\left(\frac{d y}{d x}\right)^{2}+1
$$

we may write

$$
T \frac{d^{2} y}{d x^{2}}=\alpha \sqrt{1+\left(\frac{d y}{d x}\right)^{2}}+\beta .
$$

If $p=d y / d x$, we have

$$
\frac{d p}{a \sqrt{1+p^{2}}+b}=d x
$$

where $a=\alpha / T$ and $b=\beta / T$. The equation (2) is the differential equation of the curve. To integrate it, put $p=\tan \theta ;$ then

$$
\frac{\sec ^{2} \theta d \theta}{b+a \sec \theta}=d x, \quad \text { or } \quad \frac{d \theta}{\cos \theta(a+b \cos \theta)}=d x \text {. }
$$


By the method of partial fractions, we may write

$$
\frac{d \theta}{\cos \theta}-\frac{b d \theta}{a+b \cos \theta}=a d x \text {. }
$$

Integrating, we find

(A) $\quad \log (\sec \theta+\tan \theta)$

$$
-\frac{b}{\sqrt{b^{2}-a^{2}}} \log \left[\frac{b+a \cos \theta+\sqrt{b^{2}-a^{2}} \sin \theta}{a+b \cos \theta}\right]=a x,
$$

the constant of integration being zero if we let $x=0$ when $\theta=0$. Dividing (2) by $d y$, we may write

$$
\frac{\frac{d p}{d y}}{a \sqrt{1+p^{2}}+b}=\frac{d x}{d y}=\frac{1}{p} ; \frac{p d p}{a \sqrt{1+p^{2}}+b}=d y \text {. }
$$

To integrate this put, as before $p=\tan \theta$. Then

$$
\frac{\tan \theta \sec ^{2} \theta d \theta}{a \sec \theta+b}=d y, \quad \text { or } \quad \frac{\sec \theta[d(\sec \theta)]}{a \sec \theta+b}=d y .
$$

Integrating, we find

$$
b+a \sec \theta-b \log (b+a \sec \theta)=a^{2} y+K .
$$

If $y=y_{0}$ when $\theta=0, K=b+a-b \log (a+b)-a^{2} y_{0}$, and hence

(B) $\quad a(\sec \theta-1)+b \log \left[\frac{a+b}{b+a \sec \theta}\right]=a^{2}\left(y-y_{0}\right)$.

The equations (A) and (B) are the parametric equations of the curve, the parameter being $\theta$, the angle of inclination.

3. Alternative Forms. If $b<a$, equation (A) in its present form will involve square roots of negative quantities. But in this case there is a form of (A) which is real, and which is therefore better suited for computation. This form will now be developed.

The second term of the left member of (A) is now

$$
z=\frac{b}{\sqrt{b^{2}-a^{2}}} \log \left[\frac{b+a \cos \theta+\sqrt{b^{2}-a^{2}} \sin \theta}{a+b \cos \theta}\right],
$$


that is,

or

$$
z=\frac{b}{i \sqrt{a^{2}-b^{2}}} \log \left[\frac{b+a \cos \theta+i \sqrt{a^{2}-b^{2}} \sin \theta}{a+b \cos \theta}\right],
$$

$$
e^{\frac{i z \sqrt{a^{2}-b^{2}}}{b}}=\frac{b+a \cos \theta+i \sqrt{a^{2}-b^{2}} \sin \theta}{a+b \cos \theta}
$$

or

$$
\begin{aligned}
\cos \left(\frac{z \sqrt{a^{2}-b^{2}}}{b}\right)+i & \sin \left(\frac{z \sqrt{a^{2}-b^{2}}}{b}\right) \\
& =\frac{b+a \cos \theta+i \sqrt{a^{2}-b^{2}} \sin \theta}{a+b \cos \theta} .
\end{aligned}
$$

Equating the imaginary parts,

$$
\begin{aligned}
\sin \left(\frac{z \sqrt{a^{2}-b^{2}}}{b}\right) & =\frac{\sqrt{a^{2}-b^{2}} \sin \theta}{a+b \cos \theta} \\
z & =\frac{b}{\sqrt{a^{2}-b^{2}}} \sin ^{-1}\left[\frac{\sqrt{a^{2}-b^{2}} \sin \theta}{a+b \cos \theta}\right]
\end{aligned}
$$

so that (A) may be replaced by the equation

(C) $\log (\sec \theta+\tan \theta)$

$$
-\frac{b}{\sqrt{a^{2}-b^{2}}} \sin ^{-1}\left[\frac{\sqrt{a^{2}-b^{2}} \sin \theta}{a+b \cos \theta}\right]=a x .
$$

4. Bridge Density Zero. If the linear density of the bridge becomes zero, the equations reduce to those of the common catenary. Here $b=0$, so (A) takes the simple form $\log (\sec \theta+\tan \theta)=a x$, since the second term reduces to zero. Hence we may write

$e^{a x}=\sec \theta+\sqrt{\sec ^{2} \theta-1}, \quad$ or $\quad \sec \theta=\frac{1}{2}\left(e^{a x}+e^{-a x}\right)$.

The equation (B) becomes sec $\theta-1=a\left(y-y_{0}\right)$, since the argument of the logarithm never becomes infinite. Combining these results, we have

$$
\frac{1}{2}\left(e^{a x}+e^{-a x}\right)=1+a\left(y-y_{0}\right),
$$

which is the equation of the common catenary. 
5. Cable Density Zero. When we put the linear density of the cable equal to zero, a becomes zero, and this makes (A) and (B) reduce to identities. We can, however, obtain the equations which should result. If we divide (A) throughout by $a$ and then put $a=0$, the result is an indeterminate form, of the type zero divided by zero. This is evaluated by the rule of de L'Hospital:

$$
\begin{aligned}
\left\{b /\left(a^{2}-b^{2}\right)\right\} & \left\{\left[\sqrt{b^{2}-a^{2}}\left(b+a \cos \theta+\sqrt{b^{2}-a^{2}} \sin \theta\right)\right.\right. \\
& \left.-(a+b \cos \theta)\left(\sqrt{b^{2}-a^{2}} \cos \theta-a \sin \theta\right)\right] \div \\
& {\left[\left(b+a \cos \theta+\sqrt{b^{2}-a^{2}} \sin \theta\right)(a+b \cos \theta)\right]+} \\
& \left.\frac{a}{\sqrt{b^{2}-a^{2}}} \log \left[\frac{b+a \cos \theta+\sqrt{b^{2}-a^{2}} \sin \theta}{a+b \cos \theta}\right]\right\} .
\end{aligned}
$$

When we now put $a=0$, this reduces to $(1 / b) \tan \theta$, and (A) takes the form

$$
\tan \theta=b x .
$$

Similarly, when we divide (B) through by $a^{2}$ and take the quotient of the derivatives, we have

$$
\left.\frac{(\sec \theta-1)\left(1-\frac{b^{2}}{(a+b)(b+a \sec \theta)}\right)}{2 a}\right|_{a=0}=\frac{0}{0} .
$$

Applying the rule a second time,

$$
\left.\frac{b^{2}}{2}(\sec \theta-1) \frac{b(1+\sec \theta)+2 a \sec \theta}{(a+b)^{2}(b+a \sec \theta)^{2}}\right|_{a=0}=\frac{1}{2 b} \tan ^{2} \theta,
$$

so that (B) takes the form

$$
\frac{1}{2 b} \tan ^{2} \theta=y-y_{0} \text {. }
$$

Combining $\left(\mathrm{A}^{1}\right)$ and $\left(\mathrm{B}^{1}\right)$, we may write

$$
y-y_{0}=\frac{b}{2} x^{2}
$$

which is the equation of a parabola.

The University of Chicago 